\title{
Is Neuraxial Anesthesia Safe in Patients Undergoing Surgery for Treatment of Periprosthetic Joint Infection?
}

\author{
Mohammad R. Rasouli MD, Priscilla K. Cavanaugh MS, \\ Camilo Restrepo MD, Hasan Huseyin Celyan MD, \\ Mitchell G. Maltenfort PhD, Eugene R. Viscusi MD, \\ Javad Parvizi MD
}

Received: 18 June 2014/ Accepted: 28 January 2015/Published online: 11 February 2015

(C) The Association of Bone and Joint Surgeons $\mathbb{R} 2015$

\begin{abstract}
Background There is concern that neuraxial anesthesia in patients undergoing surgery for treatment of a periprosthetic joint infection (PJI) may increase the risk of having a central nervous system infection develop. However, the available data on this topic are limited and contradictory.
\end{abstract}

One author certifies that he (JP) has or may receive payments or benefits, during the study period, an amount of USD 10,000-USD 100,000 , from Zimmer (Warsaw, IN, USA); an amount of USD 10,000-USD 100,000 from Smith \& Nephew (Andover, MA, USA); an amount less than USD 10,000 from ConvaTec (Skillman, NJ, USA); an amount of USD 10,000 from TissueGene (Rockville, MD, USA); an amount less than USD 10,000 from CeramTec (Plochingen Germany); an amount of USD 10,000 from Medtronic (Minneapolis, MN, USA); and has stock options with CD Diagnostics (Wynnewood, PA, USA), Hip Innovation Technology (Plantation, FL, USA), and PRN (Plymouth Meeting, PA, USA) that might pose a conflict of interest in connection with the submitted article.

All ICMJE Conflict of Interest Forms for authors and Clinical Orthopaedics and Related Research ${ }^{\mathbb{R}}$ editors and board members are on file with the publication and can be viewed on request. Clinical Orthopaedics and Related Research ${ }^{\mathbb{R}}$ neither advocates nor endorses the use of any treatment, drug, or device. Readers are encouraged to always seek additional information, including FDA-approval status, of any drug or device prior to clinical use. Each author certifies that his or her institution approved the human protocol for this investigation, that all investigations were conducted in conformity with ethical principles of research, and that informed consent for participation in the study was obtained.

M. R. Rasouli, P. K. Cavanaugh, C. Restrepo,

H. H. Celyan, M. G. Maltenfort, J. Parvizi

Rothman Institute of Orthopaedics, Thomas Jefferson Hospital,

Philadelphia, PA, USA

E. R. Viscusi

Department of Anesthesiology, Thomas Jefferson University,

Philadelphia, PA, USA
Questions/purposes We wished to determine whether neuraxial anesthesia (1) is associated with central nervous system infections in patients undergoing surgery for a PJI, and (2) increases the likelihood of systemic infection in these patients.

Methods All 539 patients who received neuraxial or general anesthesia during 1499 surgeries for PJI from October 2000 to May 2013 were included in this study; of these, $51 \%(n=764)$ of the surgeries were performed in 134 patients receiving neuraxial anesthesia and $49 \%$ were performed in 143 patients receiving general anesthesia. Two hundred sixty-two patients received general and neuraxial anesthesia during different surgeries. We used the International Classification of Diseases, $9^{\text {th }}$ Revision codes and the medical records to identify patients who had an intraspinal abscess or meningitis develop after surgery for a PJI. Multivariate analysis was used to assess the effect of type of anesthesia (neuraxial versus general) on postoperative complications.

Results There were no cases of meningitis, but one epidural abscess developed in a patient after neuraxial anesthesia. This patient underwent six revision surgeries during a 42-day period. Patients who received neuraxial anesthesia had lower odds of systemic infections (4\% versus 12\%; odds ratio, 0.35 ; 95\% CI, 023-054; $\mathrm{p}<0.001)$.

Conclusions Central nervous system infections after neuraxial anesthesia in patients with a PJI appear to be

J. Parvizi ( $\square)$

Rothman Institute at Thomas Jefferson University, Sheridan

Building, 10th Floor, 125 South 9th Street, Philadelphia,

PA 19107, USA

e-mail: research@rothmaninstitute.com; parvj@aol.com 
exceedingly rare. Based on the findings of this study, it may be time for the anesthesiology community to reevaluate the risk of sepsis as a relative contraindication to the use of neuraxial anesthesia.

Level of Evidence Level III, therapeutic study.

\section{Introduction}

A nationwide database analyzing 51,345 THA revision procedures showed that infection was the third most common reason for revision after THA, responsible for approximately $15 \%$ of THA revisions in the United States [4]. Furthermore, infection is the most common cause of revision after TKA [3]. Assuming that the proportion of total joint arthroplasties (TJA) complicated by infection remains unchanged, as the demand for TJAs increases, there will be a corresponding increase in the prevalence of periprosthetic joint infections (PJI) [3, 4, 17].

Surgical procedures in patients with a PJI may be performed with the patient receiving general or neuraxial anesthesia. General anesthesia once was the preferred method of anesthesia for patients undergoing a TJA [6] and overall remains the most common mode of anesthesia during orthopaedic procedures [21]. Currently, regional anesthesia, more specifically neuraxial (spinal and epidural) anesthesia, has emerged as a better alternative for patients undergoing TJA. Systematic reviews of the literature and large databases have shown there are improvements in perioperative morbidity and mortality of patients undergoing TJA who receive neuraxial anesthesia compared with general anesthesia [19, 21]. Numerous benefits of neuraxial anesthesia include reduced blood loss and decreased incidence of venous thromboembolism among others [6].

Despite the advantages of neuraxial anesthesia over general anesthesia, it has a theoretical risk for central nervous system (CNS) infections that historically have led to its relative contraindication in patients with systemic or localized infections [22]. This is attributable to the possibility that subarachnoid or dural puncture can spread infection to the epidural or intrathecal space in patients with a systemic infection [27]. Individual cases of these CNS infections have been reported [1, 13]; however, the limited and at times conflicting data available do not provide a convincing argument against the use of neuraxial anesthesia in patients with infection. In a retrospective series in Sweden involving 1,260,000 spinal and 450,000 epidural anesthetics performed during a decade, Moen et al. [22] reported epidural abscess in 13 patients and meningitis in 29 . By contrast, in a multicenter prospective study involving 30,413 epidural and 40,640 spinal anesthetics, no infectious complications were reported [2].
Gritsenko et al. [11], in a retrospective study including 474 patients who underwent removal of infected prostheses under neuraxial anesthesia, reported no clinically evident epidural abscess or meningitis complications. To the best of our knowledge, there is no study comparing neuraxial anesthesia and general anesthesia regarding the rate of postoperative systemic infections in patients undergoing surgery for treatment of a PJI.

Therefore, we sought to determine whether neuraxial anesthesia (1) is associated with CNS infections in patients undergoing surgery for PJI, and (2) increases the likelihood of systemic infections in these patients.

\section{Patients and Methods}

On approval of the institutional review board, we reviewed our electronic database to identify patients who were surgically treated for PJI at our institution from October 2000 to May 2013. Patients initially were identified using the International Classification of Diseases, 9th Revision (ICD9) codes for prosthesis infection (996.66 and 996.67). Final inclusion criteria identified patients who underwent revision surgery for an infected total hip or knee prosthesis after receiving either neuraxial anesthesia or general anesthesia. There were no children or adolescents (younger than 18 years) in the cohort. Infections were determined based on clinical signs of infection, serology including erythrocyte sedimentation rate and C-reactive protein, synovial fluid analysis, and culture results [23]. We identified 1499 total surgeries for PJIs in 539 patients. All 1499 surgeries were included in the final analysis.

Patients with a superficial surgical site infection and those who underwent revision surgery for indications other than infection were excluded. The entries were separated into two groups: patients who received general anesthesia and those who received neuraxial anesthesia. Records indicating combined general and neuraxial anesthesia and records without indication of type of anesthesia were excluded. Of the 1499 surgeries, 51\% $(\mathrm{n}=764)$ were performed in 134 patients who received neuraxial anesthesia and $49 \%(\mathrm{n}=735)$ were performed in 143 patients who received general anesthesia. Two hundred sixty-two patients received neuraxial and general anesthesia during different surgeries.

Patient demographics included age, sex, BMI, comorbidities, and American Society of Anesthesiologists (ASA) classification (Table 1). The data show the baseline comorbidity index score was higher in patients receiving general anesthesia. The comorbidities (congestive heart failure, diabetes mellitus, dementia, renal disease, chronic pulmonary disease, connective tissue disorder, prior myocardial infarction, liver disease, malignancies, peripheral 
Table 1. Patient baseline demographics based on number of surgeries

\begin{tabular}{|c|c|c|c|}
\hline Demographic & General anesthesia & Neuraxial anesthesia & $\mathrm{p}$ value \\
\hline Age (years) & $65 \pm 12$ & $65 \pm 11$ & 0.54 \\
\hline \multicolumn{4}{|l|}{ Sex } \\
\hline Male & $352(48 \%)$ & $373(49 \%)$ & \multirow[t]{2}{*}{0.76} \\
\hline Female & $383(52 \%)$ & $391(51 \%)$ & \\
\hline BMI $\left(\mathrm{kg} / \mathrm{m}^{2}\right)$ & $32 \pm 7$ & $32 \pm 7$ & 0.33 \\
\hline \multicolumn{4}{|c|}{ Charlson Comorbidity Index } \\
\hline$\leq 3$ & $634(86 \%)$ & $721(94 \%)$ & \multirow[t]{2}{*}{$<0.001$} \\
\hline$>3$ & $101(14 \%)$ & $43(6 \%)$ & \\
\hline \multicolumn{4}{|l|}{ ASA class } \\
\hline 1 & 0 & 0 & \multirow[t]{4}{*}{$<0.001$} \\
\hline 2 & $95(17 \%)$ & $144(24 \%)$ & \\
\hline 3 & $444(78 \%)$ & $453(74 \%)$ & \\
\hline 4 & $30(5 \%)$ & $13(2 \%)$ & \\
\hline
\end{tabular}

Values are mean $\pm \mathrm{SD}$ or mean with percentage in parentheses; ASA $=$ American Society of Anesthesiologists.

Table 2. ICD-9-CM diagnosis codes for major complications

\begin{tabular}{ll}
\hline Complications & ICD-9-CM diagnosis codes \\
\hline Epidural abscess & 324.1 \\
Meningitis & $320.1,320.2,320.3,320.9$ \\
Systemic infection & $038.49,038.9,041.6,041.7,785.52$, \\
& $790.7,995.91,995.92,999.31$ \\
\hline
\end{tabular}

vascular disease, cerebrovascular disease, and hemiplegia) of each patient were documented, and overall comorbidity burden was assessed using the method described by Deyo et al. [9]. The total comorbidity index was not adjusted for age.

To answer the question regarding whether neuraxial anesthesia increased the risk of infection, we queried our complication database that is collected prospectively based on the ICD-9 codes for meningitis, epidural abscess, and other postoperative complications (Table 2). Major postoperative complications analyzed included systemic infections (sepsis and septic shock).

\section{Statistical Analysis}

Baseline characteristics in the neuraxial anesthesia and general anesthesia groups were compared using Fisher's exact test for categorical variables and Wilcoxon tests for continuous variables. Logistic regressions were performed to determine if type of anesthesia (neuraxial anesthesia versus general anesthesia) was an independent predictor of postoperative complication when controlling for potential confounders. A model was created for systemic infections. All statistical analyses were performed using $\mathrm{R}$ version 3.0
(R Foundation for Statistical Computing, Vienna Austria), and probability values less than 0.05 were considered statistically significant.

\section{Results}

No patient in this cohort had meningitis develop. However, there were six patients with a possible epidural abscess. Further chart review showed that the spinal abscesses in five of these patients were not related to neuraxial anesthesia during the surgery for treatment of PJI. The sixth patient had a THA performed in 2006 followed by six revision surgeries in 2009, two under neuraxial and four under general anesthesia that all occurred within 42 days. Six days after the last revision surgery for which the patient received general anesthesia, the diagnosis of a T12-L3 epidural abscess was made. Diagnosis of an epidural abscess was made 36 days after the last revision surgery for which the patient had neuraxial anesthesia. The abscess was drained and the patient was discharged from the hospital in good condition.

After controlling for potential confounding variables, neuraxial anesthesia was associated with lower risk of systemic infection (4\% versus $12 \%$; odds ratio, $0.35 ; 95 \%$ CI, 0.23-0.54; p < 0.001) (Table 3).

\section{Discussion}

Neuraxial anesthesia improves perioperative outcome and reduces mortality compared with general anesthesia in patients who have had a TJA [12, 15, 16, 20, 21]. However, we are aware of only one study comparing neuraxial 
Table 3. Predictors of systemic infections based on the multivariate analysis

\begin{tabular}{lllr}
\hline Predictors & Odds ratio & $95 \%$ CI & p value \\
\hline Neuraxial anesthesia & 0.35 & $0.23-0.54$ & $<0.001$ \\
Year of surgery & 0.92 & $0.85-1.00$ & 0.04 \\
BMI & 1.04 & $1.02-1.06$ & $<0.001$ \\
\hline
\end{tabular}

anesthesia versus general anesthesia in terms of postoperative infection complications in patients with a PJI [11].

Our study had some limitations. Almost all patients with a PJI are treated at our institution using two-stage exchange arthroplasty including resection of the prosthesis in the first operation and reimplantation of new prosthesis in the second operation. Because some cases in our study were actually reimplantation surgeries that were part of twostage revisions, they theoretically were infection-free cases. Although this could have led to lower complication rates than if our cohort included only patients with infections, we believe this limitation is unlikely to have changed the main finding of this study in that CNS infection in patients undergoing surgical treatment of a PJI under neuraxial anesthesia is exceedingly rare. Moreover, because complications and comorbidities were identified using ICD-9-CM codes, there is a possibility for coding errors. However, coding errors exist throughout the data, likely equally distributing between the neuraxial anesthesia and general anesthesia groups. To minimize the effect of coding errors in this study, we double-checked patient charts to confirm CNS infections and all other major postoperative complications. Patients receiving general anesthesia had a higher comorbidity index as measured by ASA and Charlson comorbidity score at baseline compared with the patients receiving neuraxial anesthesia. Therefore, multivariate analysis was used to analyze the data and control for this baseline difference. Another potential limitation is the possible loss of patients with CNS infections who might have been admitted to other hospitals and thus were missed in our study. However, usually CNS infections after neuraxial anesthesia rapidly progress and will manifest during the same hospital admission or soon after and are highly likely to be detected. Finally, given the extremely low incidence of CNS infections after neuraxial anesthesia, the study might be underpowered.

The absence of patients without meningitis and the single patient who had an epidural abscess in our neuraxial anesthesia cohort supports the notion that the incidence of CNS infection is extremely rare in patients with a PJI; thus, neuraxial anesthesia can be considered safe during surgery for a PJI. However, one important factor to consider is that the majority of patients with systemic infections and almost all with a confirmed PJI are likely to receive systemic antibiotics before surgery. Prior initiation of antibiotic therapy might confer some protective role against the development of CNS-related infections. Therefore, perhaps the adjunctive use of systemic antibiotics should be required for patients receiving neuraxial anesthesia for surgical treatment of the infection. The paucity of literature regarding the safety of neuraxial anesthesia during surgery for a PJI is important in a society where the numbers of THAs and TKAs are increasing annually [17]. Although their study did not involve PJIs, Horlocker et al. [14] reported that CNS infections after neuraxial anesthesia are extremely rare. In a retrospective review of 4767 spinal anesthetics administered, only two infectious complications were found. Similarly, Auroy et al., in a multicenter prospective study including 30,413 epidural and 40,640 spinal anesthetics, found no infectious complications [2]. The results of our retrospective study suggest that the risk of a CNS infection (specifically meningitis or epidural abscess) after administration of neuraxial anesthesia for surgical treatment of a PJI of the hip or knee is extremely low. These findings parallel those of the few earlier studies $[10,16]$ evaluating the risk of CNS infection after administration of neuraxial anesthesia in patients with a systemic infection. Kotzé et al. [16] retrospectively examined the incidence of infectious complications of the epidural space in pediatric patients with empyema after receiving thoracic epidural anesthesia and none had a CNS infection develop. Another study evaluated patients with chorioamnionitis who received neuraxial anesthesia during surgery and found no cases of subsequent CNS infection [10]. A study specifically evaluating the incidence of CNS infections after surgical treatment of PJI in patients who received neuraxial anesthesia found that none of the 474 patients had subsequent meningitis or an epidural abscess [11]. Only one of our patients who received neuraxial anesthesia had a subsequent epidural abscess 6 days after surgery for a PJI, and this patient had received neuraxial anesthesia during multiple procedures. We believe that the epidural abscess in this patient was not related to the neuraxial block since it occurred more than a month after the last administration of neuraxial anesthesia. An epidural abscess after receiving neuraxial anesthesia usually progresses rapidly and manifests a few days after the neuraxial block. However, we reported this case to show that multiple administrations of neuraxial anesthesia in a short period should be done cautiously as they might increase the 
risk of a CNS infection. Administering neuraxial anesthesia multiple times to one patient might inherently increase the chance for a technical error. A technical error can arise from exposure to multiple punctures in a short time ultimately increasing the risk for an epidural abscess regardless of the presence of a PJI [8]. Therefore, when there is a history of a patient receiving neuraxial anesthesia for multiple surgeries, the decision to use neuraxial anesthesia should be made with caution.

We not only examined the incidence of CNS infection after administration of neuraxial anesthesia, but also compared general and neuraxial anesthesia in this special population to determine whether neuraxial anesthesia is associated with a lower risk of systemic infections. The effect of neuraxial anesthesia on the rate of postoperative infections, particularly surgical site infections, is contradictory [6, 7, 18]. Liu et al. [18] and Chang et al. [6], using two large databases respectively, found that neuraxial anesthesia was associated with lower risks of systemic and surgical site infections compared with general anesthesia. Neuraxial anesthesia may decrease the risk of a surgical site infection via transfusion, which has been shown to be a risk factor for surgical site infections [24]. However, the lower risk of systemic infections after using neuraxial anesthesia cannot be fully explained by reduction in transfusions and might be related to changes in immune system response after general and neuraxial anesthesia [5, 25, 26]. Volk et al. [26] suggested that neuraxial anesthesia preserves lymphocyte functions, which may contribute to "infectious resistance" after neuraxial anesthesia. However, the exact mechanism of the protective effect of neuraxial anesthesia against systemic infections is unknown and additional study is required to elucidate the mechanism.

Among the 539 patients included in our study, none had meningitis develop and only one had an epidural abscess develop after receiving neuraxial anesthesia, and that patient had undergone six revision procedures within 42 days, two of which were performed with the patient receiving neuraxial anesthesia. We found there was a lower risk of systemic infections in patients receiving neuraxial anesthesia than general anesthesia for their PJI procedures, although on balance these patients were somewhat healthier based on Charlson Comorbidity Index scores. These findings may support the notion that the incidence of CNS infection is extremely rare after neuraxial anesthesia during surgery for a PJI; thus, neuraxial anesthesia can be considered safe during this surgery. The benefits that neuraxial anesthesia confers to patients combined with the negligible risk of CNS infections with the use of this mode of anesthesia in patients with a PJI suggest that it may be time for the anesthesiology community to revisit the validity of sepsis as a relative contraindication to the use of neuraxial anesthesia.

\section{References}

1. Alpantaki K, Papoutsidakis A, Katonis P, Hadjipavlou A. Vertebral osteomyelitis, epidural and psoas abscess after epidural catheter use. Acta Orthop Belg. 2007;73:670-673.

2. Auroy Y, Narchi P, Messiah A, Litt L, Rouvier B, Samii K. Serious complications related to regional anesthesia: results of a prospective survey in France. Anesthesiology. 1997;87:479-486.

3. Bozic KJ, Kurtz SM, Lau E, Ong K, Chiu V, Vail TP, Rubash HE, Berry DJ. The epidemiology of revision total knee arthroplasty in the United States. Clin Orthop Relat Res. 2010;468:45-51.

4. Bozic KJ, Kurtz SM, Lau E, Ong K, Vail TP, Berry DJ. The epidemiology of revision total hip arthroplasty in the United States. J Bone Joint Surg Am. 2009;91:128-133.

5. Brand JM, Frohn C, Luhm J, Kirchner H, Schmucker P. Early alterations in the number of circulating lymphocyte subpopulations and enhanced proinflammatory immune response during opioid-based general anesthesia. Shock. 2003;20:213-217.

6. Chang CC, Lin HC, Lin HW, Lin HC. Anesthetic management and surgical site infections in total hip or knee replacement: a population-based study. Anesthesiology. 2010;113:279-284.

7. Curry CS, Smith KA, Allyn JW. Evaluation of anesthetic technique on surgical site infections (SSIs) at a single institution. J Clin Anesth. 2014;26:601-605.

8. Darouiche RO. Spinal epidural abscess. $N$ Engl J Med. 2006;355:2012-2020.

9. Deyo RA, Cherkin DC, Ciol MA. Adapting a clinical comorbidity index for use with ICD-9-CM administrative databases. J Clin Epidemiol. 1992;45:613-619.

10. Goodman EJ, DeHorta E, Taguiam JM. Safety of spinal and epidural anesthesia in parturients with chorioamnionitis. Reg Anesth. 1996;21:436-441.

11. Gritsenko K, Marcello D, Liguori GA, Jules-Elysée K, Memtsoudis SG. Meningitis or epidural abscesses after neuraxial block for removal of infected hip or knee prostheses. Br J Anaesth. 2012;108:485-490.

12. Guay J. The effect of neuraxial blocks on surgical blood loss and blood transfusion requirements: a meta-analysis. J Clin Anesth. 2006;18:124-128.

13. Halaby T, Leyssius A, Veneman T. Fatal bacterial meningitis after spinal anaesthesia. Scand J Infect Dis. 2007;39:280-283.

14. Horlocker TT, McGregor DG, Matsushige DK, Schroeder DR, Besse JA. A retrospective review of 4767 consecutive spinal anesthetics: central nervous system complications. Perioperative Outcomes Group. Anesth Analg. 1997;84:578-584.

15. Hu S, Zhang ZY, Hua YQ, Li J, Cai ZD. A comparison of regional and general anaesthesia for total replacement of the hip or knee: a meta-analysis. J Bone Joint Surg Br. 2009;91:935-942.

16. Kotzé A, Hinton W, Crabbe DC, Carrigan BJ. Audit of epidural analgesia in children undergoing thoracotomy for decortication of empyema. Br J Anaesth. 2007;98:662-666.

17. Kurtz S, Ong K, Lau E, Mowat F, Halpern M. Projections of primary and revision hip and knee arthroplasty in the United States from 2005 to 2030. J Bone Joint Surg Am. 2007;89:780785 .

18. Liu J, Ma C, Elkassabany N, Fleisher LA, Neuman MD. Neuraxial anesthesia decreases postoperative systemic infection risk compared with general anesthesia in knee arthroplasty. Anesth Analg. 2013;117:1010-1016. 
19. Macfarlane AJ, Prasad GA, Chan VW, Brull R. Does regional anaesthesia improve outcome after total hip arthroplasty? A systematic review. Br J Anaesth. 2009;103:335-345.

20. Mauermann WJ, Shilling AM, Zuo Z. A comparison of neuraxial block versus general anesthesia for elective total hip replacement: a meta-analysis. Anesth Analg. 2006;103:1018-1025.

21. Memtsoudis SG, Sun X, Chiu YL, Stundner O, Liu SS, Banerjee $S$, Mazumdar M, Sharrock NE. Perioperative comparative effectiveness of anesthetic technique in orthopedic patients. Anesthesiology. 2013;118:1046-1058.

22. Moen V, Dahlgren N, Irestedt L. Severe neurological complications after central neuraxial blockades in Sweden 1990-1999. Anesthesiology. 2004;101:950-959.

23. Parvizi J, Zmistowski B, Berbari EF, Bauer TW, Springer BD, Della Valle CJ, Garvin KL, Mont MA, Wongworawat MD, Zalavras CG. New definition for periprosthetic joint infection: from the Workgroup of the Musculoskeletal Infection Society. Clin Orthop Relat Res. 2011;469:2992-2994.

24. Rasouli MR, Gomes LS, Parsley B, Barsoum W, Bezwada H, Cashman J, Garcia J, Hamilton W, Hume E, Malhotra R, Memtsoudis S, Ong A, Orozco F, Padgett D, Reina R, Teloken M, Thienpont E, Waters JH. Blood conservation. J Orthop Res. 2014;32(suppl 1):S81-S89.

25. Salo M. Effects of anaesthesia and surgery on the immune response. Acta Anaesthesiol Scand. 1992;36:201-220.

26. Volk T, Schenk M, Voigt K, Tohtz S, Putzier M, Kox WJ. Postoperative epidural anesthesia preserves lymphocyte, but not monocyte, immune function after major spine surgery. Anesth Analg. 2004;98:1086-1092.

27. Wedel DJ, Horlocker TT. Regional anesthesia in the febrile or infected patient. Reg Anesth Pain Med. 2006;31:324333. 